\title{
A NOTE ON THE BOUNDEDNESS OF RIESZ TRANSFORM FOR SOME SUBELLIPTIC OPERATORS
}

\author{
FABRICE BAUDOIN AND NICOLA GAROFALO
}

\begin{abstract}
Let $\mathbb{M}$ be a smooth connected non-compact manifold endowed with a smooth measure $\mu$ and a smooth locally subelliptic diffusion operator $L$ satisfying $L 1=0$, and which is symmetric with respect to $\mu$. We show that if $L$ satisfies, with a non negative curvature parameter $\rho_{1}$, the generalized curvature inequality in (2.9) below, then the Riesz transform is bounded in $L^{p}(\mathbb{M})$ for every $p>1$, that is

$$
\left\|\sqrt{\Gamma\left((-L)^{-1 / 2} f\right)}\right\|_{p} \leq C_{p}\|f\|_{p}, \quad f \in C_{0}^{\infty}(\mathbb{M}),
$$

where $\Gamma$ is the carré du champ associated to $L$. Our results apply in particular to all Sasakian manifolds whose horizontal Tanaka-Webster Ricci curvature is nonnegative, all Carnot groups with step two, and wide subclasses of principal bundles over Riemannian manifolds whose Ricci curvature is nonnegative.
\end{abstract}

\section{Contents}

1. Introduction

2. Background

2.1. Assumptions

2.2. Some known results

3. Riesz transform

3.1. The case $1<p \leq 2$

3.2. The case $p>2$

4. Pointwise gradient estimates of the heat kernel

References

\section{INTRODUCTION}

A central result in the analysis of $\mathbb{R}^{n}$ is the $L^{p}$ continuity of singular integrals in the range $1<p<\infty$. One basic consequence of this result is the $L^{p}$ boundedness of the Riesz transforms $\mathcal{R}_{j}=\frac{\partial}{\partial x_{j}}(-\Delta)^{-1 / 2}, j=1, \ldots, n$, with their vector-valued counterpart

$$
\mathcal{R}=\left(\mathcal{R}_{1}, \ldots, \mathcal{R}_{n}\right)=\nabla(-\Delta)^{-1 / 2},
$$

see $[\mathrm{S}]$. In $[\mathrm{Str}]$ Strichartz asked the question whether such $L^{p}$ continuity of the Riesz transform could be extended to non-compact Riemannian manifolds under suitable assumptions on the latter. In this context the analogue of the vector-valued Riesz transform is the operator

$$
\mathcal{R}=\nabla \Delta^{-1 / 2}
$$

where we have denoted by $\Delta$ the Laplacian on $\mathbb{M}$ in its realization as a positive self-adjoint operator on $L^{2}(\mathbb{M})$. Strichartz's question is important for the purpose of developing analysis on

First author supported in part by NSF Grant DMS-0907326.

Second author supported in part by NSF Grant DMS-1001317. 
manifolds. To explain this point let us indicate by $(\cdot, \cdot)$ the inner product in $L^{2}(\mathbb{M})$, and with $\Delta^{1 / 2}$ the positive self-adjoint square root of $\Delta$. Then one has the equality

$$
(\Delta f, f)=\left(\Delta^{1 / 2} f, \Delta^{1 / 2} f\right) .
$$

This immediately gives

$$
\||\nabla f|\|_{2}=\left\|\Delta^{1 / 2} f\right\|_{2},
$$

which in turn allows to identify the first-order Sobolev subspaces of $L^{2}(\mathbb{M})$ obtained by completion of $C_{0}^{\infty}(\mathbb{M})$ with respect to the seminorms $\||\nabla f|\|_{2}$ and $\left\|\Delta^{1 / 2} f\right\|_{2}$. Let us also notice in passing that the latter equality can be reformulated in terms of $\mathcal{R}$ as follows

$$
\|\mathcal{R} f\|_{2}=\|f\|_{2} .
$$

However, when $1<p<\infty$ and $p \neq 2$, a similar identification of the two Sobolev spaces of order one obtained by completion of $C_{0}^{\infty}(\mathbb{M})$ with respect to the seminorms $\||\nabla f|\|_{p}$ and $\left\|\Delta^{1 / 2} f\right\|_{p}$ is no longer such a simple matter. It is a well-known fact that an estimate such as

$$
A_{p}\left\|\Delta^{1 / 2} f\right\|_{p} \leq\|\mid \nabla f\|_{p} \leq B_{p}\left\|\Delta^{1 / 2} f\right\|_{p}, \quad f \in C_{0}^{\infty}(\mathbb{M}),
$$

would suffice for such identification. It is also known that the validity of the right-hand inequality in (1.1) for a certain $1<p<\infty$ implies that of the left-hand inequality in $L^{p^{\prime}}(\mathbb{M})$, where $\frac{1}{p}+\frac{1}{p}^{\prime}=1$.

Now the right-hand inequality in (1.1) is equivalent to the $L^{p}$ continuity of the Riesz operator $\mathcal{R}$. It is then clear that (1.1) is true for all $1<p<\infty$ if

$$
\|\mathcal{R} f\|_{p} \leq C_{p}\|f\|_{p}, \quad f \in C_{0}^{\infty}(\mathbb{M})
$$

for $1<p<\infty$, and this clarifies the relevance of the question raised by Strichartz.

An interesting result due to Bakry $[\mathrm{B}$ ] states that if the Ricci curvature of $\mathbb{M}$ is bounded from below by a non negative constant then (1.2), and therefore (1.1) hold for every $1<p<\infty$. The purpose of the present note is to extend this result to a sub-Riemannian framework by using the generalized curvature-dimension inequality recently introduced by the authors in [BG1].

This extension has been recently become possible thanks to a combination of the theory developed in the two papers [BG1, [BBG], with the remarkable results in [CD, $\mathrm{ACDH}$. The latter two works have established that (1.2) does hold in the range $1<p<\infty$ for complete, noncompact Riemannian manifolds satisfying suitable general assumptions which will be discussed below. In $[\mathrm{CD}$ the authors have proved that (1.2) is true when $1<p \leq 2$. In the paper [ACDH] the authors have established (1.2) in the remaining range $2 \leq p<\infty$. The essential new contribution of the present note is to verify that such general assumptions are verified (in a non-trivial manner) for a general class of locally subelliptic operators satisfying on a given smooth manifold $\mathbb{M}$ the generalized curvature-dimension inequality $\operatorname{CD}\left(\rho_{1}, \rho_{2}, \kappa, d\right)$ in (2.9) below, with curvature parameter $\rho_{1} \geq 0$ (this in the Riemannian case corresponds to Ric $\geq 0$ ). Once this is done, the $L^{p}$ continuity of an appropriately defined Riesz operator will follow by the general real variable methods developed in [CD], $\mathrm{ACDH}]$.

To state the main result in this paper we assume that $\mathbb{M}$ be a $C^{\infty}$ connected, non-compact manifold endowed with a smooth measure $\mu$. Throughout the paper, the notation $L^{p}(\mathbb{M})$, $1 \leq p \leq \infty$, indicates the space of $p$-summable functions on $\mathbb{M}$ with respect to the measure $\mu$. We assume that on $\mathbb{M}$ a second-order diffusion operator $L$ with real coefficients is given. We also suppose that $L$ be locally subelliptic, non-positive, and that it satisfy the assumptions listed in Section 2. There is a natural notion of (square of the length of the) "gradient" associated with 
$L$, namely

$$
\Gamma(f)=\frac{1}{2}\left\{L\left(f^{2}\right)-2 f L f\right\},
$$

and a canonical distance $d$, see (2.4) below. We assume throughout that the metric space $(\mathbb{M}, d)$ be complete. We also suppose that $\mathbb{M}$ be endowed with another bilinear differential form $\Gamma^{Z}$, see (2.6) below, and that $\Gamma$ and $\Gamma^{Z}$ satisfy all the hypothesis in Section 2 below. From our perspective, the most significant assumption is the so-called generalized curvature-dimension inequality $\operatorname{CD}\left(\rho_{1}, \rho_{2}, \kappa, d\right)$ in (2.9) below, which we now recall for the reader's convenience:

There exist constants $\rho_{1} \geq 0, \rho_{2}>0, \kappa \geq 0$, and $d \geq 2$ such that the inequality

$$
\Gamma_{2}(f)+\nu \Gamma_{2}^{Z}(f) \geq \frac{1}{d}(L f)^{2}+\left(\rho_{1}-\frac{\kappa}{\nu}\right) \Gamma(f)+\rho_{2} \Gamma^{Z}(f)
$$

hold for every $f \in C^{\infty}(\mathbb{M})$ and every $\nu>0$, where $\Gamma_{2}$ and $\Gamma_{2}^{Z}$ are defined by (2.7) and (2.8) below.

The assumption (1.3) constitutes a sub-Riemannian generalization of the classical curvaturediemension inequality $\mathrm{CD}(\rho, n)$

$$
\Gamma_{2}(f) \geq \frac{1}{n}(\Delta f)^{2}+\rho \Gamma(f),
$$

which, as a consequence of the well-known Bochner's identity, is known to hold on any $n$ dimensional Riemannian manifold satisfying Ric $\geq \rho$.

The parameter $\rho_{1}$ in $\operatorname{CD}\left(\rho_{1}, \rho_{2}, \kappa, d\right)$ has the meaning of a lower bound on a sub-Riemannian Ricci tensor, see [BG1] for extensive details. Throughout the present paper the assumption $\rho_{1} \geq 0$ will be in force. The semigroup $P_{t}=e^{t L}$ is a strongly continuous semigroup of contraction operators on $L^{p}(\mathbb{M})$ for $1 \leq p \leq \infty$. We denote by $p(x, y, t)=p(y, x, t)$ the positive heat kernel on $\mathbb{M}$ associated with the semigroup $P_{t}$. Given $f \in C_{0}^{\infty}(\mathbb{M})$, the function

$$
u(x, t)=P_{t} f(x)=\int_{M} f(y) p(x, y, t) d \mu(y),
$$

is a solution of the equation $L u-u_{t}=0$ in $\mathbb{M} \times(0, \infty)$, corresponding to the initial datum $u(x, 0)=f(x), x \in \mathbb{M}$.

We now recall that, in the general framework described above, in the paper [BG1] we proved a generalized Li-Yau type inequality for solutions of the heat equation on $\mathbb{M}$ of the form $u=P_{t} f$. From such inequality, we were able to derive several basic facts, among which the following off-diagonal Gaussian upper bound: For any $0<\varepsilon<1$ there exists a constant $C\left(\rho_{2}, \kappa, d, \varepsilon\right)>0$, which tends to $\infty$ as $\varepsilon \rightarrow 0^{+}$, such that for every $x, y \in \mathbb{M}$ and $t>0$ one has

$$
p(x, y, t) \leq \frac{C\left(d, \kappa, \rho_{2}, \varepsilon\right)}{V(x, \sqrt{t})^{\frac{1}{2}} V(y, \sqrt{t})^{\frac{1}{2}}} \exp \left(-\frac{d(x, y)^{2}}{(4+\varepsilon) t}\right) .
$$

Hereafter in this paper we adopt the notation

$$
V(x, r)=\mu(B(x, r)),
$$

where for $x \in \mathbb{M}$ and $r>0$ we have let $B(x, r)=\{y \in \mathbb{M} \mid d(y, x)<r\}$.

In the paper BBG] we further developed the program initiated in BG1 and were able to obtain the following basic result: There exists a constant $C_{d}>0$ depending only on $\rho_{1}, \rho_{2}, \kappa, d$, such that for every $x \in \mathbb{M}$ and $r>0$ one has

$$
V(x, 2 r) \leq C_{d} V(x, r) .
$$


For a purely analytical proof of (1.5) in the Riemannian setting we refer the reader to the paper BG2].

Now in their work [CD the authors proved that the two results (1.4) and (1.5) are enough to establish the weak- $(1,1)$ continuity of the Riesz transforms for the space of homogeneous type $(\mathbb{M}, d)$. Since from integration by parts, and from the identity $(L f, f)=\left\|(-L)^{1 / 2} f\right\|_{2}$ the strong $L^{2}$ continuity of the Riesz transform

$$
\left\|\sqrt{\left.\Gamma\left((-L)^{1 / 2}\right) f\right)}\right\|_{2}=\|f\|_{2}
$$

trivially follows, by the Marcinckiewicz interpolation theorem we thus obtain the following result.

Theorem 1.1. Let $1<p \leq 2$. There is a constant $C_{p}>0$ such that for every $f \in C_{0}^{\infty}(\mathbb{M})$,

$$
\left\|\sqrt{\Gamma\left((-L)^{-1 / 2} f\right)}\right\|_{L^{p}(\mathbb{M})} \leq C_{p}\|f\|_{L^{p}(\mathbb{M})} .
$$

Theorem 1.1 provides the $L^{p}$ continuity of the absolute value of the Riesz operator

$$
T f=\sqrt{\Gamma\left((-L)^{-1 / 2} f\right)}
$$

within the range $1<p \leq 2$. We emphasize is that $T$ is a sublinear operator. For the remaining range $2 \leq p<\infty$ we appeal to the real variable theory developed in the work [ACDH]. We recall the salient ingredients of the general approach in that paper:

1) $e^{t L} 1=1$ (stochastic completeness);

2) global doubling condition;

3) global Poincaré inequality;

4) Caccioppoli type inequalities;

5) Gaffney type estimates;

6) bounds for $\sqrt{t} \sqrt{\Gamma\left(e^{t L}\right)}$.

As for 1) the stochastic completeness in our framework follows as a special case of Theorem 3.5 in [BG1, see also $\mathrm{Mu}$ ] for an extension of such result. Regarding 2) we have already discussed (1.5). As for 3), we mention that in [BBG] it was proved that there exists $C_{p}>0$, depending only on $\rho_{1}, \rho_{2}, \kappa, d$, such that for every $x \in \mathbb{M}$ and $r>0$ one has

$$
\int_{B(x, r)}\left|f-f_{B}\right|^{2} d \mu \leq C_{p} r^{2} \int_{B(x, r)} \Gamma(f) d \mu
$$

for every $f \in C^{1}(\bar{B}(x, r))$. Thus 3 . is available to us.

We are thus missing ingredients 4), 5) and 6) In this note we establish these results, see Corollary 3.5. Lemmas 3.6, 3.7 and 3.10, and Theorem 4.1 below. This allows us to close the circle and, by using the work $\mathrm{ACDH}$, obtain the following result.

Theorem 1.2. Let $2 \leq p<\infty$. There is a constant $C_{p}>0$ such that for every $f \in C_{0}^{\infty}(\mathbb{M})$,

$$
\left\|\sqrt{\Gamma\left((-L)^{-1 / 2} f\right)}\right\|_{L^{p}(\mathbb{M})} \leq C_{p}\|f\|_{L^{p}(\mathbb{M})} .
$$

By combining Theorems 1.1 and 1.2 we obtain the following result.

Theorem 1.3. Let $1<p<\infty$. There exist constants $A_{p}, B_{p}>0$ such that

$$
A_{p}\left\|(-L)^{1 / 2} f\right\|_{p} \leq\|\sqrt{\Gamma(f)}\|_{p} \leq B_{p}\left\|(-L)^{1 / 2} f\right\|_{p}, \quad f \in C_{0}^{\infty}(\mathbb{M}),
$$


The results in this paper establish the continuity of the Riesz transform and the equivalence of the Sobolev spaces defined by completion of $C_{0}^{\infty}(\mathbb{M})$ with respect to the two seminorms in (3.2) for the various classes of sub-Riemannian manifolds which are encompassed by the general framework of BG1]. While we refer the reader to that source for a detailed discussion of the examples, here we confine ourselves to mention the following basic result which is a corollary of our work.

Theorem 1.4. Let $(\mathbb{M}, \theta)$ be a $\mathrm{CR}$ manifold with real dimension $2 n+1$ and vanishing TanakaWebster torsion, i.e., a Sasakian manifold. If there exists $\rho_{1} \geq 0$ such that for every $x \in \mathbb{M}$ the Tanaka-Webster Ricci tensor satisfies the bound

$$
\operatorname{Ric}_{x}(v, v) \geq \rho_{1}|v|^{2},
$$

for every horizontal vector $v \in \mathcal{H}_{x}$, then given any $1<p<\infty$ the Riesz transform associated with a sub-Laplacian on $\mathbb{M}$ is continuous on $L^{p}(\mathbb{M})$.

In connection with Theorem 1.4 we mention that it was proved in BG1 that in the framework of Theorem 1.4 the generalized curvature-dimension inequality $\operatorname{CD}\left(\rho_{1}, \rho_{2}, \kappa, d\right)$ does hold with $\rho_{1} \geq 0$. Thus these manifolds fall within the scope of the assumptions in Section 2 .

In closing, we mention some known partial results related to those in the present paper. In [LV] the boundedness of the Riesz transforms was proved on every stratified nilpotent Lie group. In A] this result was generalized to Lie groups of polynomial growth.

Acknowledgment: The second named author would like to thank Steve Hofmann for several helpful discussions.

\section{BACKGROUND}

2.1. Assumptions. Hereafter in this paper, $\mathbb{M}$ will be a $C^{\infty}$ connected and non-compact manifold endowed with a smooth measure $\mu$. Throughout the paper, the notation $L^{p}(\mathbb{M}), 1 \leq p \leq \infty$, indicates the space of $p$-summable functions on $\mathbb{M}$ with respect to the measure $\mu$.

We assume that on $M$ a second-order diffusion operator $L$ with real coefficients is given. We also suppose that $L$ be locally subelliptic (for the relevant definition and properties of such operators see [FSC] and [JSC]), and that it satisfy:

1) $L 1=0$

2) $\int_{\mathbb{M}} f L g d \mu=\int_{\mathbb{M}} g L f d \mu$;

3) $\int_{\mathbb{M}} f L f d \mu \leq 0$,

for every $f, g \in C_{0}^{\infty}(\mathbb{M})$.

There is a natural gradient (or rather, a natural square of the length of a gradient) canonically associated with $L$, and it is given by the quadratic functional $\Gamma(f)=\Gamma(f, f)$, where

$$
\Gamma(f, g)=\frac{1}{2}(L(f g)-f L g-g L f), \quad f, g \in C^{\infty}(\mathbb{M}) .
$$

The functional $\Gamma(f)$ is known as le carré du champ. Notice that $\Gamma(1)=0$. Furthermore, using the results in [PS], locally in the neighborhood of every point $x \in \mathbb{M}$ we can write

$$
L=-\sum_{i=1}^{m} X_{i}^{*} X_{i},
$$

where the vector fields $X_{i}$ are Lipschitz continuous (such representation is not unique, but this fact is of no consequence for us. We note for further reference that the number $m$ of vector fields entering in the local representation (2.2) is bounded above by the dimension of the manifold 
$\mathbb{M})$. Therefore, for any $x \in \mathbb{M}$ there exists an open neighborhood $U_{x}$ such that in $U_{x}$ we have for any $f \in C^{\infty}(\mathbb{M})$

$$
\Gamma(f)=\sum_{i=1}^{m}\left(X_{i} f\right)^{2} .
$$

This shows that $\Gamma(f) \geq 0$ and it actually only involves differentiation of order one. Furthermore, the value of $\Gamma(f)(x)$ does not depend on the particular representation (2.2) of $L$. With the operator $L$ we can also associate a canonical distance:

$$
d(x, y)=\sup \left\{|f(x)-f(y)| \mid f \in C^{\infty}(\mathbb{M}),\|\Gamma(f)\|_{\infty} \leq 1\right\}, \quad x, y \in \mathbb{M},
$$

where for a function $g$ on $\mathbb{M}$ we have let $\|g\|_{\infty}=\underset{\mathbb{M}}{\operatorname{ess} \sup }|g|$.

A tangent vector $v \in T_{x} \mathbb{M}$ is called subunit for $L$ at $x$ if $v=\sum_{i=1}^{m} a_{i} X_{i}(x)$, with $\sum_{i=1}^{m} a_{i}^{2} \leq 1$, see [FP]. It turns out that the notion of subunit vector for $L$ at $x$ does not depend on the local representation (2.2) of $L$. A Lipschitz path $\gamma:[0, T] \rightarrow \mathbb{M}$ is called subunit for $L$ if $\gamma^{\prime}(t)$ is subunit for $L$ at $\gamma(t)$ for a.e. $t \in[0, T]$. We then define the subunit length of $\gamma$ as $\ell_{s}(\gamma)=T$. Given $x, y \in \mathbb{M}$, we indicate with

$$
S(x, y)=\{\gamma:[0, T] \rightarrow \mathbb{M} \mid \gamma \text { is subunit for } L, \gamma(0)=x, \gamma(T)=y\} .
$$

In this paper we assume that

$$
S(x, y) \neq \varnothing, \quad \text { for every } x, y \in \mathbb{M} .
$$

Under such assumption it is easy to verify that

$$
d_{s}(x, y)=\inf \left\{\ell_{s}(\gamma) \mid \gamma \in S(x, y)\right\},
$$

defines a true distance on $\mathbb{M}$. Furthermore, thanks to Lemma 5.43 in [CKS] we know that

$$
d(x, y)=d_{s}(x, y), \quad x, y \in \mathbb{M},
$$

hence we can work indifferently with either one of the distances $d$ or $d_{s}$.

In addition to the differential form (2.1), we assume that $\mathbb{M}$ be endowed with another smooth bilinear differential form, indicated with $\Gamma^{Z}$, satisfying for $f, g \in C^{\infty}(\mathbb{M})$

$$
\Gamma^{Z}(f g, h)=f \Gamma^{Z}(g, h)+g \Gamma^{Z}(f, h),
$$

and $\Gamma^{Z}(f)=\Gamma^{Z}(f, f) \geq 0$. Given the first-order bilinear forms $\Gamma$ and $\Gamma^{Z}$ on $\mathbb{M}$, we now introduce the following second-order differential forms:

$$
\begin{gathered}
\Gamma_{2}(f, g)=\frac{1}{2}[L \Gamma(f, g)-\Gamma(f, L g)-\Gamma(g, L f)], \\
\Gamma_{2}^{Z}(f, g)=\frac{1}{2}\left[L \Gamma^{Z}(f, g)-\Gamma^{Z}(f, L g)-\Gamma^{Z}(g, L f)\right] .
\end{gathered}
$$

Observe that if $\Gamma^{Z} \equiv 0$, then $\Gamma_{2}^{Z} \equiv 0$ as well. As for $\Gamma$ and $\Gamma^{Z}$, we will use the notations $\Gamma_{2}(f)=\Gamma_{2}(f, f), \Gamma_{2}^{Z}(f)=\Gamma_{2}^{Z}(f, f)$.

We make the following assumptions that will be in force throughout the paper:

(H.1) There exists an increasing sequence $h_{k} \in C_{0}^{\infty}(\mathbb{M})$ such that $h_{k} \nearrow 1$ on $\mathbb{M}$, and

$$
\left\|\Gamma\left(h_{k}\right)\right\|_{\infty}+\left\|\Gamma^{Z}\left(h_{k}\right)\right\|_{\infty} \rightarrow 0, \quad \text { as } k \rightarrow \infty .
$$

(H.2) For any $f \in C^{\infty}(\mathbb{M})$ one has

$$
\Gamma\left(f, \Gamma^{Z}(f)\right)=\Gamma^{Z}(f, \Gamma(f)) .
$$


(H.3) The generalized curvature-dimension inequality $C D\left(\rho_{1}, \rho_{2}, \kappa, d\right)$ be satisfied with $\rho_{1} \geq 0$, that is: There exist constants $\rho_{1} \geq 0, \rho_{2}>0, \kappa \geq 0$, and $d \geq 2$ such that the inequality

$$
\Gamma_{2}(f)+\nu \Gamma_{2}^{Z}(f) \geq \frac{1}{d}(L f)^{2}+\left(\rho_{1}-\frac{\kappa}{\nu}\right) \Gamma(f)+\rho_{2} \Gamma^{Z}(f)
$$

hold for every $f \in C^{\infty}(\mathbb{M})$ and every $\nu>0$, where $\Gamma_{2}$ and $\Gamma_{2}^{Z}$ are defined by 2.7 and 2.8 .

For example, the assumptions (H.1)-(H.3) are satisfied in all Carnot groups of step two, and in all complete Sasakian manifolds whose horizontal Tanaka-Webster Ricci curvature is non negative. For further examples, including a wide class of bundles over Riemannian manifolds we refer the reader to BG1].

In this framework:

- $L$ is essentially self-adjoint on $C_{0}^{\infty}(\mathbb{M})$, so that by using the spectral theorem for the Friedrichs extension of $L$ in the Hilbert space $L^{2}(\mathbb{M})$, we may construct a strongly continuous contraction semigroup $\left(P_{t}\right)_{t \geq 0}$ in $L^{2}(\mathbb{M})$ whose infinitesimal generator is $L$;

- By hypoellipticity of $L,\left(P_{t}\right)_{t \geq 0}$ admits a heat kernel, that is: There is a smooth function $p(t, x, y), t \in(0, \infty), x, y \in \mathbb{M}$, such that for every $f \in L^{2}(\mathbb{M})$ and $x \in \mathbb{M}$,

$$
P_{t} f(x)=\int_{\mathbb{M}} p(t, x, y) f(y) d \mu(y) .
$$

Moreover, the heat kernel satisfies the two following conditions:

(i) (Symmetry) $p(t, x, y)=p(t, y, x)$;

(ii) (Chapman-Kolmogorov relation) $p(t+s, x, y)=\int_{\mathbb{M}} p(t, x, z) p(s, z, y) d \mu(z)$;

- The semigroup $\left(P_{t}\right)_{t \geq 0}$ is a sub-Markovian semigroup: If $0 \leq f \leq 1$ is a function in $L^{2}(\mathbb{M})$, then $0 \leq P_{t} f \leq 1$

- By the Riesz-Thorin interpolation theorem, $\left(P_{t}\right)_{t \geq 0}$ defines a contraction semigroup on $L^{p}(\mathbb{M}), 1 \leq p \leq \infty$.

2.2. Some known results. In this section we collect some results that, under the above listed assumptions, were proved in the works [BG1] and [BBG]. Such results constitute the backbone of the present paper.

The first basic result is that the manifold $\mathbb{M}$ is stochastically complete with respect to $L$, i.e., for every $t>0$

$$
P_{t} 1=e^{t L} 1=1 \text {. }
$$

We recall that this result is equivalent to the uniqueness of the bounded solution of the Cauchy problem

$$
\left\{\begin{array}{l}
L u-u_{t}=0, \quad \mathbb{M} \times(0, \infty), \\
u(x, 0)=\varphi(x), \quad x \in \mathbb{M},
\end{array}\right.
$$

with bounded initial datum $\varphi$. The property (2.10) is a corollary of Theorem 3.5 in [BG1], see also $[\mathrm{Mu}]$ for an extension of such result.

Another basic result is the following Gaussian upper bound that was proved in [BG1].

Theorem 2.1. For any $0<\varepsilon<1$ there exists a constant $C(\varepsilon)=C\left(d, \kappa, \rho_{2}, \varepsilon\right)>0$, which tends to $\infty$ as $\varepsilon \rightarrow 0^{+}$, such that for every $x, y \in \mathbb{M}$ and $t>0$ one has

$$
p(x, y, t) \leq \frac{C(\varepsilon)}{V(x, \sqrt{t})} \exp \left(-\frac{d(x, y)^{2}}{(4+\varepsilon) t}\right) .
$$

In the paper $[\mathrm{BBG}$ it has been proved that the metric measure space $(\mathbb{M}, d, \mu)$ satisfies the global volume doubling property and that, furthermore, the $L^{2}$ Poincaré inequality is satisfied on balls. More precisely, we have the following result. 
Theorem 2.2. There exist constants $C_{d}, C_{p}>0$, depending only on $\rho_{1}, \rho_{2}, \kappa, d$, for which one has for every $x \in \mathbb{M}$ and every $r>0$ :

$$
\begin{aligned}
V(x, 2 r) & \leq C_{d} V(x, r) \\
\int_{B(x, r)}\left|f-f_{B}\right|^{2} d \mu & \leq C_{p} r^{2} \int_{B(x, r)} \Gamma(f) d \mu,
\end{aligned}
$$

for every $f \in C^{1}(\bar{B}(x, r))$.

We list for future use the following well-known consequence of the doubling condition (2.11).

Corollary 2.3. With $C_{d}$ as in (2.11) define

$$
Q=\log _{2} C_{d} .
$$

Then, for every $x \in \mathbb{M}$ and any $0<r<R<\infty$ one has

$$
V(x, R) \leq C_{d}\left(\frac{R}{r}\right)^{Q} V(x, r) .
$$

In particular, if $y, z \in \mathbb{M}$ and $t>0$ we have

$$
V(y, \sqrt{t}) \leq C_{d}\left(\frac{d(y, z)}{\sqrt{t}}+1\right)^{Q} V(z, \sqrt{t})
$$

and also for any given $\alpha>0$, there exists a constant $C>0$ depending on $C_{d}$ and $\alpha$, such that

$$
\int_{\mathbb{M}} \exp \left(-\alpha \frac{d(y, z)^{2}}{t}\right) d \mu(z) \leq C V(y, \sqrt{t}) .
$$

Proof. The proof of (2.13) is standard and we omit it. As for (2.14) it is enough to apply (2.13) with $R=d(y, z)+\sqrt{t}$ and $r=\sqrt{t}$, to obtain

$$
V(y, \sqrt{t}) \leq V(z, d(y, z)+\sqrt{t}) \leq C_{d}\left(\frac{d(y, z)+\sqrt{t}}{\sqrt{t}}\right)^{Q} V(z, \sqrt{t}) .
$$

Finally, (2.15) easily follows by covering $\mathbb{M}$ with dyadic rings $B\left(y, 2^{k+1} \sqrt{t}\right) \backslash B\left(y, 2^{k} \sqrt{t}\right)$, and then using (2.11).

\section{RIESZ TRANSFORM}

Our objective in this section is proving that the Riesz transform associated to $L$ is bounded in $L^{p}(\mathbb{M})$. 1

Theorem 3.1. Let for every $1<p<\infty$. There is a constant $C_{p}>0$ such that for every $f \in L^{p}(\mathbb{M})$,

$$
\left\|\sqrt{\Gamma\left((-L)^{-1 / 2} f\right)}\right\|_{L^{p}(\mathbb{M})} \leq C_{p}\|f\|_{L^{p}(\mathbb{M})} .
$$

As a consequence of Theorem 3.1, we obtain the following result.

Theorem 3.2. Let $1<p<\infty$. There exist constants $A_{p}, B_{p}>0$ such that

$$
A_{p}\left\|(-L)^{1 / 2} f\right\|_{p} \leq\|\sqrt{\Gamma(f)}\|_{p} \leq B_{p}\left\|(-L)^{1 / 2} f\right\|_{p}, \quad f \in C_{0}^{\infty}(\mathbb{M}) .
$$

\footnotetext{
${ }^{1}$ In the case where $\mu(\mathbb{M})<\infty$ one has to consider the space $L_{0}^{p}(\mathbb{M})$ of functions in $L^{p}(\mathbb{M})$ with mean 0 , see $[\mathrm{ACDH}]$; this modification will be implicit in the text.
} 
3.1. The case $1<p \leq 2$. Following our discussion in the introduction, the boundedness of the Riesz transform on $L^{p}(\mathbb{M})$ for $1<p \leq 2$ follows by combining Theorem 4.1 and (2.11) in Theorem 2.2 above with Theorem 1.1 in [CD]. We note explicitly that (2.11) implies that $(M, d, \mu)$ is a space of homogeneous type according to [CW], see also [C], and so all tools of real analysis are available. From these results the weak- $(1,1)$ continuity of the Riesz transform

$$
\mu\left(\left\{x \in \mathbb{M} \mid \sqrt{\left.\Gamma\left((-L)^{-1 / 2} f\right)(x)\right)}>\lambda\right\}\right) \leq \frac{C}{\lambda}\|f\|_{L^{1}(\mathbb{M})}, \quad \lambda>0,
$$

can be established as in $[\mathrm{CD}$. Then, for the range $1<p \leq 2$ the inequality (3.1) follows by applying Marcinckiewicz real interpolation theorem. The reader should notice that the operator

$$
T=\sqrt{\left.\Gamma\left((-L)^{-1 / 2} f\right)\right)}
$$

is a sublinear operator, i.e., $|T(f+g)(x)| \leq|T f(x)|+|T g(x)|$ for every $f, g$ and a.e. $x \in \mathbb{M}$.

3.2. The case $p>2$. Following the general method in the proof of Theorem 3.1 in [ACDH], to establish the boundedness of the Riesz transform when $p>2$, we need to the ingredients listed as 1)-6) in the introduction. As it was mentioned there the items which are at this point missing are Caccioppoli and Gaffney type estimates, as well as bounds for $\sqrt{t} \sqrt{\Gamma\left(e^{t L}\right)}$. This section is devoted to filling this gap. We begin with establishing the former type of result.

3.2.1. Caccioppoli type estimates. In what follows we prove an a priori inequality of Caccioppoli type for the heat semigroup $P_{t}$.

Proposition 3.3. Let $f \in C_{0}^{\infty}(\mathbb{M})$. For $t \geq 0$ we have

$$
\Gamma\left(P_{t} f\right)+\rho_{2} t \Gamma^{Z}\left(P_{t} f\right) \leq \frac{1+\frac{2 \kappa}{\rho_{2}}}{2 t}\left(P_{t}\left(f^{2}\right)-\left(P_{t} f\right)^{2}\right) .
$$

Proof. Let us fix $T>0$. Given a function $f \in C_{0}(\mathbb{M})$, for $0 \leq t \leq T$ we introduce the functionals

$$
\begin{gathered}
\phi_{1}(x, t)=\Gamma\left(P_{T-t} f\right)(x), \\
\phi_{2}(x, t)=\Gamma^{Z}\left(P_{T-t} f\right)(x),
\end{gathered}
$$

which are defined on $\mathbb{M} \times[0, T]$. It is is easy to check that, with $\Gamma_{2}$ and $\Gamma_{2}^{Z}$ defined as in (2.7) and (2.8), we have

$$
L \phi_{1}+\frac{\partial \phi_{1}}{\partial t}=2 \Gamma_{2}\left(P_{T-t} f\right)
$$

and

$$
L \phi_{2}+\frac{\partial \phi_{2}}{\partial t}=2 \Gamma_{2}^{Z}\left(P_{T-t} f\right)
$$

see also [BG1]. Consider now the function

$$
\begin{aligned}
\phi(x, t) & =a(t) \phi_{1}(x, t)+b(t) \phi_{2}(x, t) \\
& =a(t) \Gamma\left(P_{T-t} f\right)(x)+b(t) \Gamma^{Z}\left(P_{T-t} f\right)(x),
\end{aligned}
$$

where $a$ and $b$ are two nonnegative functions that will be chosen later. At this point we observe that, since by hypothesis $\rho_{1} \geq 0$, the generalized curvature-dimension $\operatorname{CD}\left(\rho_{1}, \rho_{2}, \kappa, d\right)$ in (2.9) trivially implies the generalized curvature-dimension inequality $\operatorname{CD}\left(0, \rho_{2}, \kappa, \infty\right)$. Applying the latter with the choice $\nu=\frac{b}{a}$ we thus obtain

$$
\begin{aligned}
L \phi+\frac{\partial \phi}{\partial t} & =a^{\prime} \Gamma\left(P_{T-t} f\right)+b^{\prime}\left(P_{T-t} f\right) \Gamma^{Z}\left(P_{T-t} f\right)+2 a \Gamma_{2}\left(P_{T-t} f\right)+2 b\left(P_{T-t} f\right) \Gamma_{2}^{Z}\left(P_{T-t} f\right) \\
& \geq\left(a^{\prime}-2 \kappa \frac{a^{2}}{b}\right) \Gamma\left(P_{T-t} f\right)+\left(b^{\prime}+2 \rho_{2} a\right) \Gamma^{Z}\left(P_{T-t} f\right) .
\end{aligned}
$$


Let us now chose

$$
a(t)=\frac{1}{\rho_{2}}(T-t), \quad b(t)=(T-t)^{2} .
$$

With this choice we have on $[0, T]$,

$$
b^{\prime}+2 \rho_{2} a \equiv 0,
$$

and

We find then

$$
a^{\prime}-2 \kappa \frac{a^{2}}{b}=-\frac{1}{\rho_{2}}-\frac{2 \kappa}{\rho_{2}^{2}} .
$$

$$
L \phi+\frac{\partial \phi}{\partial t} \geq\left(-\frac{1}{\rho_{2}}-\frac{2 \kappa}{\rho_{2}^{2}}\right) \Gamma\left(P_{T-t} f\right) .
$$

From a comparison theorem for parabolic partial differential equations (see for instance p.52 in [F] or Proposition 3.2 in [BG1]) we deduce

$$
P_{T}(\phi(\cdot, T))(x) \geq \phi(x, 0)-\left(\frac{1}{\rho_{2}}+\frac{2 \kappa}{\rho_{2}^{2}}\right) \int_{0}^{T} P_{t}\left(\Gamma\left(P_{T-t} f\right)\right) d t .
$$

To conclude, we consider the functional

$$
\left.\Psi(t)=\frac{1}{2} P_{t}\left(\left(P_{T-t} f\right)^{2}\right)\right) .
$$

A straightforward computation shows that

$$
\begin{aligned}
\Psi^{\prime}(t) & \left.=\frac{1}{2} P_{t}\left(L\left(P_{T-t} f\right)^{2}\right)\right)+P_{t}\left(P_{T-t} f \frac{\partial}{\partial t}\left(P_{T-t} f\right)\right) \\
& \left.=\frac{1}{2} P_{t}\left(L\left(P_{T-t} f\right)^{2}\right)-2 P_{T-t} f L\left(P_{T-t} f\right)\right)=P_{t}\left(\Gamma\left(P_{T-t} f\right)\right) .
\end{aligned}
$$

This gives

$$
\int_{0}^{T} P_{t}\left(\Gamma\left(P_{T-t} f\right) d t=\Psi(T)-\Psi(0)=\frac{1}{2}\left(P_{T}\left(f^{2}\right)-\left(P_{T} f\right)^{2}\right) .\right.
$$

Replacing this information in (3.3), along with the identities

$$
\phi(x, 0)=\frac{1}{\rho_{2}} T \Gamma\left(P_{T} f\right)+T^{2} \Gamma^{Z}\left(P_{T} f\right), \quad \phi(x, T)=0,
$$

we reach the desired conclusion.

As a corollary of Proposition 3.3 and of the $L^{p}$ continuity of $P_{t}$, for $1 \leq p \leq \infty$, we obtain the following Caccioppoli type estimates.

Corollary 3.4. For any $f \in C_{0}^{\infty}(\mathbb{M}), 2 \leq p \leq \infty$ we have

$$
\left\|\sqrt{\Gamma\left(P_{t} f\right)}\right\|_{p} \leq \sqrt{\frac{1+\frac{2 \kappa}{\rho_{2}}}{2 t}}\|f\|_{p} .
$$

In what follows we will also need the following result.

Corollary 3.5. Let $n$ be the dimension of $\mathbb{M}$. With $C=\sqrt{\frac{\left(2 \kappa+\rho_{2}\right) n}{2 \rho_{2}}}$ we have

$$
\sup _{x \in \mathbb{M}} \int_{\mathbb{M}} \sqrt{\Gamma(p(\cdot, y, t))(x)} d \mu(y) \leq \frac{C}{\sqrt{t}} .
$$

This estimate implies that for any $f \in L^{\infty}(\mathbb{M})$,

$$
\left\|\sqrt{\Gamma\left(P_{t} f\right)}\right\|_{\infty} \leq \frac{C}{\sqrt{t}}\|f\|_{\infty}
$$


Proof. For $x \in \mathbb{M}$ fixed, let then $U_{x}$ be a sufficiently small neighborhood of $x$ in which we can write $L$ as in (2.2), where the vector fields $X_{i}$ are Lipschitz continuous. For $f \in C_{0}^{\infty}(\mathbb{M})$, we have

$$
\left|\int_{\mathbb{M}} X_{i} p(\cdot, y, t)(x) f(y) d \mu(y)\right|=\left|X_{i} P_{t} f(x)\right| \leq\left\|\sqrt{\Gamma\left(P_{t} f\right)}\right\|_{\infty} \leq \sqrt{\frac{1+\frac{2 \kappa}{\rho_{2}}}{2 t}}\|f\|_{\infty}
$$

Let $\varepsilon>0$ and take now

$$
f(y)=h_{k}(y) \frac{X_{i} p(\cdot, y, t)(x)}{\left|X_{i} p(\cdot, y, t)(x)\right|+\varepsilon},
$$

where $0 \leq h_{k} \leq 1$ is an increasing sequence in $C_{0}^{\infty}(\mathbb{M})$, converging to 1 . We obtain

$$
\int_{\mathbb{M}} h_{k}(y) \frac{X_{i} p(\cdot, y, t)(x)^{2}}{\left|X_{i} p(\cdot, y, t)(x)\right|+\varepsilon} d \mu(y) \leq \sqrt{\frac{1+\frac{2 \kappa}{\rho_{2}}}{2 t}}
$$

By the monotone convergence theorem and by Fatou's theorem we deduce, first letting $k \rightarrow \infty$ and then $\varepsilon \rightarrow 0$, that

$$
\int_{\mathbb{M}}\left|X_{i} p(\cdot, y, t)(x)\right| d \mu(y) \leq \sqrt{\frac{1+\frac{2 \kappa}{\rho_{2}}}{2 t}} .
$$

The estimate (3.4) follows immediately from the latter inequality.

3.2.2. Gaffney-type estimates. We now turn to the second main ingredient which are Gaffney type estimates. In what follows we indicate with $E, F \subset \mathbb{M}$ two closed subsets. We need the following results.

Lemma 3.6. For every two closed sets $E, F \subset \mathbb{M}$, and any $f \in L^{\infty}(\mathbb{M})$ supported in $E$, one has

$$
\left\|P_{t} f\right\|_{L^{2}(F)} \leq e^{-\frac{d(E, F)^{2}}{4 t}}\|f\|_{L^{2}(E)} .
$$

Proof. Let us suppose $d(E, F)>0$, otherwise the conclusion follows trivially from the $L^{2}$ continuity of $P_{t}$. As a first step we let $\psi$ denote a function in $\operatorname{Lip}(\mathbb{M})$, such that $\Gamma(\psi) \leq 1$ a.e. on M. With $\alpha>0$ and $\phi=e^{-\alpha \psi}$, we note that

$$
\Gamma(\phi)=\alpha^{2} \phi^{2} \Gamma(\psi) \leq \alpha^{2} \phi^{2} .
$$

Let $f \in L^{\infty}(\mathbb{M})$, and consider

$$
y(t)=\left\|\phi P_{t} f\right\|_{L^{2}(\mathbb{M})}^{2} .
$$

Denoting with $\left\langle\cdot, \cdot>\right.$ the inner product in $L^{2}(\mathbb{M})$, we have

$$
\begin{aligned}
y^{\prime}(t) & =2<L\left(P_{t} f\right), \phi^{2} P_{t} f>=-2 \int_{\mathbb{M}} \Gamma\left(P_{t} f, \phi^{2} P_{t} f\right) d \mu \\
& =-2 \int_{\mathbb{M}} \phi^{2} \Gamma\left(P_{t} f\right) d \mu-4 \int_{\mathbb{M}} \phi P_{t} f \Gamma\left(P_{t} f, \phi\right) d \mu \\
& \leq-2 \int_{\mathbb{M}} \phi^{2} \Gamma\left(P_{t} f\right) d \mu+\frac{2}{\varepsilon} \int_{\mathbb{M}} \phi^{2} \Gamma\left(P_{t} f\right) d \mu+2 \varepsilon \alpha^{2} \int_{\mathbb{M}} \phi^{2}\left(P_{t} f\right)^{2} d \mu,
\end{aligned}
$$

where to estimate the last term we have used (3.6). Choosing $\varepsilon=1$ we conclude

$$
y^{\prime}(t) \leq 2 \alpha^{2} y(t)
$$

and, upon integrating this inequality, we obtain

$$
\left\|\phi P_{t} f\right\|_{L^{2}(\mathbb{M})} \leq e^{\alpha^{2} t}\|\phi f\|_{L^{2}(\mathbb{M})} .
$$


We now want to show that (3.7) yields the desired conclusion. Suppose that $\operatorname{supp} f \subset E$, then we argue as follows. We take $\psi(x)=d(x, F)$, and for any $\alpha>0$ we let $\phi=e^{-\alpha \psi}$. Since $\phi \equiv 1$ on $F$, from (3.7) we obtain

$$
\left\|P_{t} f\right\|_{L^{2}(F)} \leq\left\|\phi P_{t} f\right\|_{L^{2}(\mathbb{M})} \leq e^{\alpha^{2} t}\|\phi f\|_{L^{2}(\mathbb{M})}=e^{\alpha^{2} t}\|\phi f\|_{L^{2}(E)} .
$$

Now, for any $x \in E$ we have $\psi(x) \geq d(E, F)$, and thus $\phi \leq e^{-\alpha d(E, F)}$ on $E$. This gives

$$
\left\|P_{t} f\right\|_{L^{2}(F)} \leq e^{\alpha^{2} t-\alpha d(E, F)}\|f\|_{L^{2}(E)} .
$$

By choosing $\alpha=\frac{d(E, F)}{2 t}>0$ we reach the desired conclusion.

For $\omega>0$ sufficiently small denote

$$
S_{\omega}=\left\{z=r e^{i \theta} \in \mathbb{C}|0<r<\infty,| \theta \mid<\frac{\pi}{2}+\omega\right\} .
$$

Since $L$ generates an analytic semigroup $e^{z L}$ in a sector $S_{\omega}$, we have

$$
P_{t}=\frac{1}{2 \pi i} \int_{\Gamma_{\delta}} e^{t \zeta} R(\zeta ; L) d \zeta
$$

where $\Gamma_{\delta} \subset S_{\omega}$ is the path composed of the two rays $r e^{i \theta}$ and $r e^{-i \theta}$, with $0<r<\infty$ and $\frac{\pi}{2}<\theta<\frac{\pi}{2}+\delta, 0<\delta<\omega$, and $R(\zeta ; L)$ is the resolvent of $L$. Using the same argument as in Lemma 3.6] it is easy to see that the Gaffney estimate (3.7) continues to be valid for $P_{z} f$ with $z \in S_{\delta}$ for any fixed $0<\delta<\omega$. Similarly to what was done above this leads to an estimate of the type

$$
\left\|P_{z} f\right\|_{L^{2}(F)} \leq e^{\alpha^{2}|\Re z|-\alpha d(E, F)}\|f\|_{L^{2}(E)}, \quad z \in S_{\delta},
$$

for any $f \in L^{\infty}(\mathbb{M})$ which is supported in $E$, and any $\alpha>0$.

Lemma 3.7. There exists a constant $C>0$ such that for every two closed sets $E, F \subset \mathbb{M}$, and any $f \in L^{\infty}(\mathbb{M})$ supported in $E$, one has

$$
t|| L P_{t} f\left\|_{L^{2}(F)} \leq C e^{-\frac{d(E, F)^{2}}{6 t}}\right\| f \|_{L^{2}(E)} .
$$

Proof. Consider the path

$$
\Gamma_{t}=\left\{z=t\left(1+\frac{e^{i \theta}}{2}\right) \in \mathbb{C} \mid 0 \leq \theta \leq 2 \pi\right\} \subset S_{\omega} .
$$

Using the analyticity of $P_{t}$ in the sector $S_{\omega}$ we can write

$$
t \frac{\partial P_{t}}{\partial t}=\frac{t}{2 \pi i} \int_{\Gamma_{t}} \frac{e^{z L}}{(z-t)^{2}} d z=\frac{1}{\pi} \int_{0}^{2 \pi} e^{-i \theta} e^{t\left(1+\frac{e^{i \theta}}{2}\right) L} d \theta .
$$

Using (3.8), this gives

$$
\begin{aligned}
t\left\|L P_{t} f\right\|_{L^{2}(F)} & \leq \frac{1}{\pi} \int_{0}^{2 \pi}\left\|P_{t\left(1+\frac{e^{i \theta}}{2}\right)} f\right\|_{L^{2}(F)} \\
& \leq 2 e^{\alpha^{2} t\left(1+\frac{|\cos \theta|}{2}\right)-\alpha d(E, F)}\|f\|_{L^{2}(E)} \\
& \leq 2 e^{\frac{3}{2} \alpha^{2} t-\alpha d(E, F)}\|f\|_{L^{2}(E)}
\end{aligned}
$$

Choosing $\alpha=\frac{d(E, F)}{3 t}$ yields the desired conclusion. 
Since the distance function $y \rightarrow d(x, y)$ is obviously a Lipschitz continuous function on $\mathbb{M}$ (with respect to $d$ itself), in view of (2.2) and (2.3), and of a Rademacher type theorem for Lipschitz vector fields we can construct Lipschitz continuous cut-off functions on metric balls, see for instance Theorem 1.5 in [GN]. We collect this fact in the following lemma.

Lemma 3.8. Let $0<s<t<\infty$. There exists a constant $C>0$ such that for every $B(x, s) \subset$ $B(x, t) \subset \mathbb{M}$ there exists a function $\varphi \in \operatorname{Lip}(\mathbb{M})$, with $0 \leq \varphi \leq 1, \varphi \equiv 1$ on $B(x, s)$, and supp $\varphi \subset B(x, t)$, for which

$$
\sqrt{\Gamma(\varphi)} \leq \frac{C}{t-s}
$$

Corollary 3.9. Given a closed set $F \subset \mathbb{M}$, consider the open set $F_{\varepsilon}=\{x \in \mathbb{M} \mid d(x, F)<\varepsilon\}$. There exists a function $\varphi \in \operatorname{Lip}(\mathbb{M})$ such that $0 \leq \varphi \leq 1, \varphi \equiv 1$ on $F_{\varepsilon / 2}$, and $\varphi \equiv 0$ in $\mathbb{M} \backslash F_{\varepsilon}$, and for which

$$
\sqrt{\Gamma(\varphi)} \leq \frac{C}{\varepsilon}
$$

Proof. It follows from Lemma 3.8 by a standard partition of unity argument.

We are now in a position to establish the third Gaffney type estimate which we will need.

Lemma 3.10. There exist constants $C \geq 0$ and $\alpha>0$ such that for any two closed sets $E, F \subset \mathbb{M}$, and every function $f \in L^{\infty}(\mathbb{M})$ supported in $E$, one has

$$
\sqrt{t}\left\|\sqrt{\Gamma\left(P_{t} f\right)}\right\|_{L^{2}(F)} \leq C e^{-\alpha \frac{d(E, F)^{2}}{t}}\|f\|_{L^{2}(E)} .
$$

Proof. We adapt the argument on p. 930 in $\mathrm{ACDH}$. If $d(E, F) \leq \sqrt{t}$ there is nothing to prove. We can thus assume that $d(E, F)>\sqrt{t}$. With $\varepsilon=\frac{d(E, F)}{3}$, consider the set $F_{\varepsilon}$, and pick a function $\varphi \in \operatorname{Lip}(\mathbb{M})$ supported in $F_{\varepsilon}$ as in Corollary 3.9. We have

$$
\begin{aligned}
t \int_{\mathbb{M}} \varphi^{2} \Gamma\left(P_{t} f\right) d \mu & =\frac{t}{2} \int_{\mathbb{M}} \varphi^{2}\left[L\left(\left(P_{t} f\right)^{2}\right)-2 P_{t} f L\left(P_{t} f\right)\right] d \mu \\
& =-t \int_{\mathbb{M}} \varphi^{2} P_{t} f L P_{t} f d \mu-2 t \int_{\mathbb{M}} \varphi P_{t} f \Gamma\left(\varphi, P_{t} f\right) d \mu \\
& \leq t\left\|L P_{t} f\right\|_{L^{2}\left(F_{\varepsilon}\right)}\left\|P_{t} f\right\|_{L^{2}(\mathbb{M})}+2\left(t \int_{\mathbb{M}} \varphi^{2} \Gamma\left(P_{t} f\right) d \mu\right)^{1 / 2}\left(t \int_{\mathbb{M}} \Gamma(\varphi)\left(P_{t} f\right)^{2} d \mu\right)^{1 / 2} .
\end{aligned}
$$

We now use Lemma 3.7 and the $L^{2}$ continuity of $P_{t}$ to obtain for some $\alpha>0$

$$
t\left\|L P_{t} f\right\|_{L^{2}\left(F_{\varepsilon}\right)}\left\|P_{t} f\right\|_{L^{2}(\mathbb{M})} \leq C e^{-\frac{d\left(E, F_{\varepsilon}\right)^{2}}{6 t}}\|f\|_{L^{2}(E)}^{2} \leq C e^{-\alpha \frac{d(E, F)^{2}}{t}}\|f\|_{L^{2}(E)}^{2} .
$$

Recalling that $\sqrt{t}<d(E, F)$, and using the support property of $\Gamma(\varphi)$, and the estimate $\Gamma(\varphi) \leq$ $C d(E, F)^{-2}$ from Corollary [3.9, we conclude

$$
\begin{aligned}
\left(\sqrt{t} \int_{\mathbb{M}} \Gamma(\varphi)\left(P_{t} f\right)^{2} d \mu\right)^{1 / 2} & \leq C\left(\int_{F_{\varepsilon}}\left(P_{t} f\right)^{2} d \mu\right)^{1 / 2} \\
& \leq C e^{-\frac{d\left(E, F_{\varepsilon}\right)^{2}}{4 t}}\|f\|_{L^{2}(E)} \leq C e^{-\alpha \frac{d(E, F)^{2}}{t}}\|f\|_{L^{2}(E)}
\end{aligned}
$$

for an appropriate $\alpha>0$. We conclude

$$
t \int_{\mathbb{M}} \varphi^{2} \Gamma\left(P_{t} f\right) d \mu \leq C e^{-\alpha \frac{d(E, F)^{2}}{t}}\|f\|_{L^{2}(E)}^{2}+C e^{-\alpha \frac{d(E, F)^{2}}{t}}\|f\|_{L^{2}(E)}\left(t \int_{\mathbb{M}} \varphi^{2} \Gamma\left(P_{t} f\right) d \mu\right)^{1 / 2} .
$$

A trivial estimate allows to conclude that

$$
t \int_{\mathbb{M}} \varphi^{2} \Gamma\left(P_{t} f\right) d \mu \leq C e^{-\alpha \frac{d(E, F)^{2}}{t}}\|f\|_{L^{2}(E)}^{2} .
$$


Since

$$
t\left\|\sqrt{\Gamma\left(P_{t} f\right)}\right\|_{L^{2}(F)}^{2} \leq t \int_{\mathbb{M}} \varphi^{2} \Gamma\left(P_{t} f\right) d \mu
$$

we have reached the desired conclusion.

3.2.3. The completion of the proof Theorem 3.1 in the range $2 \leq p<\infty$. We are now in a position to prove Theorem 3.1 in the range $2 \leq p<\infty$. As mentioned above, all we need to do at this point is combine Theorem 2.2, Corollary 3.5 and Lemmas 3.6, 3.7 and 3.10 with the real variable results in $\underline{\mathrm{ACDH}}$.

\section{Pointwise gradient estimates of the heat kernel}

In this section we investigate the validity of pointwise gradient estimates of the heat kernel. Besides being interesting in their own right, such estimates are also connected with Theorem 3.1 in the range $2 \leq p<\infty$. For a detailed discussion of this aspect we refer the reader to [ACDH].

Theorem 4.1. There exists a constant $C=C\left(d, \kappa, \rho_{2}\right)>0$ such that for every $x, y \in \mathbb{M}$ and $t>0$ one has

$$
\sqrt{\Gamma(p(\cdot, y, t))}(x) \leq \frac{C}{\sqrt{t} V(y, \sqrt{t})} .
$$

Proof. We fix a point $x \in M$ and $s>0$ and begin with the observation that if we consider the function

$$
f_{x, s}(z)=p(x, z, s)
$$

then thanks to Theorem 4.1 there exists a constant $C>0$ (independent of $x \in \mathbb{M}$ and $s>0$ ) such that $f_{x, s} \in L^{\infty}(\mathbb{M})$ and

$$
\left\|f_{x, s}\right\|_{L^{\infty}(\mathbb{M})} \leq \frac{C}{V(x, \sqrt{s})} .
$$

We next observe that, given points $x, y \in \mathbb{M}$ and $t>0$, then we can write

$$
p(x, y, t)=P_{\frac{t}{2}}\left(f_{y, \frac{t}{2}}\right)(x) .
$$

For $x \in \mathbb{M}$ fixed, let then $U_{x}$ be a sufficiently small neighborhood of $x$ in which we can write $L$ as in (2.2). We thus have

$$
\begin{aligned}
\left|X_{i} p(\cdot, y, t)(x)\right| & =\left|X_{i}\left(P_{\frac{t}{2}}\left(f_{y, \frac{t}{2}}\right)\right)(x)\right| \leq \sqrt{\Gamma\left(P_{\frac{t}{2}}\left(f_{y, \frac{t}{2}}\right)\right)}(x) \\
& \leq\left\|\sqrt{\Gamma\left(P_{\frac{t}{2}}\left(f_{y, \frac{t}{2}}\right)\right)}\right\|_{L^{\infty}(\mathbb{M})} \leq \frac{C}{\sqrt{t}}\left\|f_{y, \frac{t}{2}}\right\|_{L^{\infty}(\mathbb{M})} \\
& \leq \frac{C}{\sqrt{t} V(y, \sqrt{t / 2})},
\end{aligned}
$$

where in the last inequality we have used the $L^{\infty}$ Caccioppoli inequality in Corollary 3.5. This estimate finally gives

$$
\sqrt{\Gamma(p(\cdot, y, t))}(x)=\sqrt{\sum_{i=1}^{d}\left(X_{i} p(\cdot, y, t)(x)\right)^{2}} \leq \frac{C}{\sqrt{t} V(y, \sqrt{t / 2})} .
$$

The proof is completed by an application of (2.11) in Theorem 2.2 , 
Theorem 4.2. For every $\varepsilon>0$ there exists a constant $C(\varepsilon)=C\left(d, \kappa, \rho_{2}, \varepsilon\right)>0$ such that for every $x, y \in \mathbb{M}$ and $t>0$ one has

$$
\sqrt{\Gamma(p(\cdot, y, t))}(x) \leq \frac{C(\varepsilon)}{\sqrt{t} V(y, \sqrt{t})} \exp \left(-\frac{d(x, y)^{2}}{4(1+\varepsilon) t}\right) .
$$

Proof. It suffices to appeal to Theorem 4.11 in CS. In such result the authors, by a beautiful use of Phragmén-Lindelöf theory, prove that the combination of (2.13) in Corollary 2.3, and of the estimates

and

$$
p(x, x, t) \leq \frac{C}{V(x, \sqrt{t})}
$$

$$
\sqrt{\Gamma(p(\cdot, y, t))}(x) \leq \frac{C}{\sqrt{t} V(y, \sqrt{t})}
$$

allows to improve the estimate in Theorem 4.1 into the following one

$$
\sqrt{\Gamma(p(\cdot, y, t))}(x) \leq \frac{C}{\sqrt{t} V(y, \sqrt{t})}\left(1+\frac{d(x, y)^{2}}{4 t}\right)^{1+3 Q} \exp \left(-\frac{d(x, y)^{2}}{4 t}\right),
$$

where $Q$ is as (2.13). From the latter estimate the desired conclusion follows.

\section{REFERENCES}

[ACDH] P. Auscher, T. Coulhon, X.T. Duong, S. Hofmann, Riesz transform on manifolds and heat kernel regularity Ann. Sci. École Norm. Sup. (4) 37 (2004), no. 6, 911-957.

[A] G. Alexopoulos, An application of homogenization theory to harmonic analysis: Harnack inequalities and Riesz transforms on Lie groups of polynomial growth. Canad. J. Math. 44 (1992), no. 4, 691-727.

[B] D. Bakry, Étude des transformations de Riesz dans les variétés riemanniennes à courbure de Ricci minorée. (French) [A study of Riesz transforms in Riemannian manifolds with minorized Ricci curvature] Séminaire de Probabilités, XXI, 137-172, Lecture Notes in Math., 1247, Springer, Berlin, 1987.

[BG1] F. Baudoin \& N. Garofalo, Curvature-dimension inequalities and Ricci lower bounds for sub-Riemannian manifolds with transverse symmetries, Arxiv preprint 1101.3590, submitted paper (2009).

[BG2] _ Perelman's entropy and doubling property on Riemannian manifolds, J. Geom. Anal., to appear.

[BBG] F. Baudoin. M. Bonnefont \& N. Garofalo, A sub-Riemannian curvature-dimension inequality, volume doubling property and the Poincaré inequality, arXiv:1007.1600, submitted, (2010).

[CKS] E. Carlen, S. Kusuoka \& D. Stroock, Upper bounds for symmetric Markov transition functions, Ann. Inst. H. Poincaré Probab. Statist. 23 (1987), no. 2, suppl., 245-287.

[C] M. Christ, A T (b) theorem with remarks on analytic capacity and the Cauchy integral, Colloq. Math. 60/61 (1990), no. 2, 601628.

[CW] R. R. Coifman \& G. Weiss, Analyse harmonique non-commutative sur certains espaces homogènes. (French) Étude de certaines intégrales singulières, Lecture Notes in Mathematics, Vol. 242. Springer-Verlag, BerlinNew York, 1971. v+160 pp.

[CD] T. Coulhon, X. T. Duong, Riesz transforms for $1 \leq p \leq 2$, Trans. Amer. Math. Soc., 3513 (1999), 1151-1169.

[CS] T. Coulhon, A. Sikora, Gaussian heat kernel upper bounds via the Phragmn-Lindelf theorem. Proc. Lond. Math. Soc. (3) 96 (2008), no. 2, 507-544.

[FP] C. Fefferman \& D. H. Phong, Subelliptic eigenvalue problems, Conference on harmonic analysis in honor of Antoni Zygmund, Vol. I, II (Chicago, Ill., 1981), 590-606, Wadsworth Math. Ser., Wadsworth, Belmont, CA, 1983.

[FSC] C. L. Fefferman \& A. Sánchez-Calle, Fundamental solutions for second order subelliptic operators, Ann. of Math. (2) 124 (1986), no. 2, 247-272.

[F] A. Friedman, Partial differential equations of parabolic type, Dover, 2008.

[GN] N. Garofalo \& D.-M. Nhieu, Lipschitz continuity, global smooth approximations and extension theorems for Sobolev functions in Carnot-Carathodory spaces, J. Anal. Math. 74 (1998), 67-97.

[JSC] D. Jerison \& A. Sánchez-Calle, Subelliptic second order differential operators, Lecture. Notes in Math., 1277 (1987), pp. 46-77. 
[LV] Lohoué, N., Varopoulos, N.: Remarques sur les transformées de Riesz sur les groupes nilpotents. C.R.A.S. Paris, 301, 11 (1985) 559-560.

$[\mathrm{Mu}]$ I. Munive, Generalized curvature-dimension inequalities, stochastic completeness and volume growth, preprint, 2011.

[PS] R. S. Phillips \& L. Sarason, Elliptic-parabolic equations of the second order, J. Math. Mech. 17 1967/1968, 891-917.

[S] E. M. Stein, Singular integrals and differentiability properties of functions, Princeton Univ. Press, 1970.

[Str] R. Strichartz, Analysis of the Laplacian on the complete Riemannian manifold, J. Funct. Anal. 52 (1983), 48-79.

Department of Mathematics, Purdue University, West Lafayette, IN 47907

E-mail address, Fabrice Baudoin: fbaudoin@math.purdue.edu

Department of Mathematics, Purdue University, West Lafayette, IN 47907

E-mail address, Nicola Garofalo: garofalo@math.purdue.edu 